\title{
LHC constraints on Yukawa unification in SO(10)
}

\author{
Marcin Badziak $^{a, b}$ and Kazuki Sakurai ${ }^{c}$ \\ ${ }^{a}$ Department of Applied Mathematics and Theoretical Physics, \\ Centre for Mathematical Sciences, University of Cambridge, \\ Wilberforce Road, Cambridge CB3 0WA, U.K. \\ ${ }^{b}$ Cavendish Laboratory, University of Cambridge, \\ J.J. Thomson Avenue, Cambridge CB3 OHE, U.K. \\ ${ }^{c}$ Deutsches Elektronen Synchrotron DESY, \\ Notkestrasse 85, D-22607 Hamburg, Germany \\ E-mail: M.Badziak@damtp.cam.ac.uk, kazuki.sakurai@desy.de
}

ABSTRACT: LHC constraints on the recently proposed SUSY SO(10) GUT model with topbottom-tau Yukawa unification are investigated. In this model, various phenomenological constraints are in concord with the Yukawa unification thanks to the negative sign of $\mu$, $D$-term splitting in the soft scalar masses and non-universal gaugino masses generated by a non-zero $F$-term in a 24-dimensional representation of $\mathrm{SU}(5) \subset \mathrm{SO}(10)$. After discussing the impact of the CP-odd Higgs boson mass bound on this model, we provide a detailed analysis of the recent direct SUSY searches performed by ATLAS and investigate the constraints on this $\mathrm{SO}(10)$ model. At $95 \%$ confidence level, the lower limit on the gluino mass is found to be $675 \mathrm{GeV}$. Assuming an integrated luminosity of $10 \mathrm{fb}^{-1}$, this bound may be extended to $1.1 \mathrm{TeV}$ if the right-handed down squark is lighter than about $1 \mathrm{TeV}$.

KEYwords: Supersymmetry Phenomenology

ArXiv EPRINT: 1112.4796 


\section{Contents}

1 Introduction 1

2 Description of the SO(10) model 3

3 ATLAS searches for squarks and gluinos $\quad 6$

4 Methodology and its validation $\quad 9$

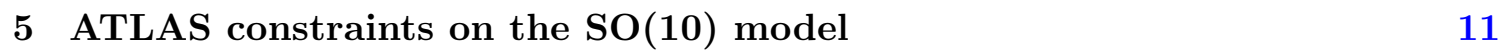

$\begin{array}{llr}6 & \text { Summary and conclusions } & 16\end{array}$

$\begin{array}{ll}\text { A Validation of our simulation } & 17\end{array}$

\section{Introduction}

In the simplest versions of supersymmetric (SUSY) Grand Unified Theories (GUTs) based on the $\mathrm{SO}(10)$ gauge symmetry, in which the Minimal Supersymmetric Standard Model (MSSM) Higgs doublets, $H_{u}$ and $H_{d}$, reside in a $\mathbf{1 0}$ of SO(10), the Yukawa couplings of top, bottom and tau unify at the GUT scale $[1,2]$. Unlike the gauge coupling unification, Yukawa coupling unification is very sensitive to weak scale threshold corrections which depend on the entire SUSY spectrum [3-5]. Moreover, top-bottom-tau Yukawa unification is often in conflict with radiative electroweak symmetry breaking (REWSB) [4]. Therefore, the condition of the top-bottom-tau Yukawa unification at the GUT scale imposes nontrivial constraints on the soft SUSY breaking terms. In particular, it has been shown that the Yukawa unification is incompatible with the REWSB in the Constrained MSSM (CMSSM) where the soft masses for scalars and gauginos are universal at the GUT scale [4].

$\mathrm{SO}(10)$ models are rather constrained not only because of the requirement of Yukawa unification but also because all the MSSM sfermions of each generation fit into a single 16 of $\mathrm{SO}(10)$. Thus, assuming the Minimal Flavor Violation [6, 7], the only non-universality in the scalar masses consistent with the $\mathrm{SO}(10)$ gauge symmetry is the mass splitting between sfermions and Higgses. This has been shown to be insufficient to make the Yukawa unification compatible with the REWSB [8]. However, if the $\mathrm{SO}(10)$ gauge symmetry is spontaneously broken, as is generally expected, in the effective theory below the GUT scale, a generic $D$-term contribution splits the scalar masses residing in the same representation of $\mathrm{SO}(10)$ [9]. Most importantly, the $D$-term contribution splits the masses of the MSSM Higgs doublets and may make the Yukawa unification consistent with the REWSB [10]. 
The requirement of the top-bottom-tau Yukawa unification prefers negative values of the Higgs-mixing parameter, $\mu .{ }^{1}$ However, a model with the negative $\mu$ and the universal gaugino masses $[11,12]$ predicts a negative SUSY contribution to the muon anomalous magnetic moment, $(g-2)_{\mu}$, and enlarges the observed discrepancy between theory and experiment. That is why most of the studies that assume the universal gaugino masses in the last decade have been devoted to the case of the positive $\mu$ [13-16]. In such a case the Yukawa unification can be realized but there is a price for it. Namely, the scalar masses are pushed to the multi- $\mathrm{TeV}$ regime so proper REWSB is obtained at the expense of severe finetuning. Moreover, these models have serious problems with the FCNC constraints $[17,18]$ because of a very large value of $\left|\mu A_{t}\right|$, in particular with a lower limit on $\operatorname{BR}\left(B_{s} \rightarrow \mu^{+} \mu^{-}\right)$ which was recently improved by the CMS and the LHCb experiments [19].

Last but not least, in that class of models the solutions with the most accurate unification of the Yukawa couplings at the GUT scale found in the numerical scans performed in ref. $[13-16,20]$ are characterised by the gluino mass of about $350 \mathrm{GeV},{ }^{2}$ while the recent ATLAS "b-jet" search [22] excluded the gluino masses up to 450 (570) GeV in a model with "ad hoc" splitting of the Higgs masses [13-15] ( $D$-term splitting of the scalar masses [16]). For a recent review of $\mathrm{SO}(10)$ models with the third family Yukawa coupling unification see ref. [23].

A remedy to the problems of the $\mathrm{SO}(10)$ models discussed above was put forward recently in ref. [24] where a novel model with negative $\mu$ was proposed. In this model the gaugino masses are assumed to be generated by an $F$-term which is a non-singlet of $\mathrm{SO}(10)$ transforming as a $\mathbf{2 4}$ of $\mathrm{SU}(5) \subset \mathrm{SO}(10)$. Such an assumption leads to the gaugino masses with the following pattern at the GUT scale: $M_{1}: M_{2}: M_{3}=-1:-3: 2$ [25]. The main phenomenological success of the model is its consistency with all experimental constraints including the $(g-2)_{\mu}$ and the $\mathrm{BR}(b \rightarrow s \gamma)$ which are satisfied thanks to the positive sign of the product $\mu M_{2}$ [26]. Another advantage of the model is the prediction of a rather light SUSY spectrum which can be tested at the LHC. In particular, the gluino mass is predicted to be in the range of about $500-700$ or $900-1550 \mathrm{GeV}$. It is not unreasonable to expect that some of the parameter space corresponding to such a light gluino has already been excluded by the LHC. However, the LHC experiments interpret their data mainly in the CMSSM and some simplified models and the obtained mass limits for SUSY particles cannot be applied directly to other models. There are several studies that reinterpret the LHC SUSY searches in terms of the SUSY models other than the CMSSM. The LHC constraints on gauge mediation [27, 28], anomaly mediation [29], phenomenological SUSY models [30, 31], SUSY models with compressed spectra [32, 33] and light third generation models [34-39] have been studied. The constraints on models with the top-bottom-tau

\footnotetext{
${ }^{1}$ We use the sign convention in which the gluino mass parameter $M_{3}$ and the gluino mass $m_{\tilde{g}}$ are positive.

${ }^{2}$ The usually dominant SUSY correction to the bottom mass is proportional to $\mu m_{\tilde{g}} / m_{\tilde{b}_{2}}^{2}$ when $m_{\tilde{b}_{2}} \gtrsim$ $m_{\tilde{g}}$. Assuming the Yukawa unification and $\mu>0$, this correction drives the bottom mass away from its experimental value so gluino is required to be much lighter than the heavier sbottom to suppress this correction. Typically this implies very light gluino in accord with findings of ref. [13-16, 20]. However, the necessity of very light gluino in the Yukawa-unified models with $\mu>0$ was questioned recently in ref. [21] where new solutions with exact Yukawa unification were found with the gluino mass as large as $1.4 \mathrm{TeV}$.
} 
Yukawa unification in the context of SUSY SU(4) $)_{c} \times \mathrm{SU}(2)_{L} \times \mathrm{SU}(2)_{R}[40,41]$ have also been investigated [42]. In the present paper we study the constraints and implications of the recent LHC data on the $\mathrm{SO}(10)$ model proposed in ref. [24]. We focus on three ATLAS analyses of the final states with large missing transverse momentum and no leptons: the "0-lepton" search with $1.04 \mathrm{fb}^{-1}$ [43], the "b-jet" search with $0.83 \mathrm{fb}^{-1}[22]$ and the "multijets" search with $1.34 \mathrm{fb}^{-1}$ of data [44].

The paper is organized as follows. In section 2 we describe in some more detail the $\mathrm{SO}$ (10) model proposed in ref. [24]. In section 3 we summarise the ATLAS searches for squarks and gluinos. In section 4 we present our methodology of simulating the ATLAS searches and its validation. Interpretation of the ATLAS searches in the $\mathrm{SO}(10)$ model proposed in ref. [24] is given in section 5 . We conclude in section 6 . In the appendix we give additional information on the validation of our simulation of the ATLAS searches.

\section{Description of the $\mathrm{SO}(10)$ model}

We start with a brief review of the $\mathrm{SO}(10)$ model proposed in ref. [24]. The first crucial feature of this model is that the Higgs-mixing parameter $\mu$ is negative. The negative sign of $\mu$ is phenomenologically preferred because it allows for the negative threshold correction to the bottom mass [3-5] (as required by the Yukawa unification) without the need to push up the scalar masses to multi-TeV regime. Even though negative $\mu$ is preferred by the Yukawa unification, it leads to discrepancy with the experimental results for the $(g-2)_{\mu}$ and the $\operatorname{BR}(b \rightarrow s \gamma)$ if the gaugino masses are universal. As explained in detail in ref. [24], phenomenologically acceptable models require $\mu M_{2}>0$. In order to satisfy this condition, the gaugino masses are assumed to be generated by a non-zero $F$-term in a 24-dimensional representation of $\mathrm{SU}(5) \subset \mathrm{SO}(10)$. This leads to the following pattern of the gaugino masses at the GUT scale [25]:

$$
\left(M_{1}, M_{2}, M_{3}\right)=\left(-\frac{1}{2},-\frac{3}{2}, 1\right) M_{1 / 2}
$$

It should be stressed that even though non-universalities in the gaugino masses are introduced, only one free parameter, $M_{1 / 2}$, governs the gaugino sector.

The soft scalar masses also need to be non-universal to achieve the Yukawa unification and light sparticle spectra. ${ }^{3}$ It is assumed that the $D$-term of the $\mathrm{U}(1)_{\mathrm{V}}(\mathrm{SO}(10) \supset$ $\left.\mathrm{SU}(5) \times \mathrm{U}(1)_{\mathrm{V}}\right)$ vector superfield acquires a vacuum expectation value at the GUT scale. ${ }^{4}$

\footnotetext{
${ }^{3}$ In ref. [45] Yukawa-unified solutions with gaugino masses given in eq. (2.1) and universal scalar masses were found. However, the price for the universality in the scalar sector is very heavy spectrum of squarks and gluinos inaccessible at the LHC. Moreover, such solutions predict too small SUSY contribution to the $(g-2)_{\mu}$ to be consistent with the experimental data. In the present paper we demand that the constraint from the $(g-2)_{\mu}$ is satisfied at least at $2 \sigma$ level.

${ }^{4}$ In ref. [46] a model with $\mu<0$ and non-universal gaugino masses given in eq. (2.1) was considered in which sfermion masses are assumed to be universal and "ad hoc" Higgs mass splitting is introduced which explicitly breaks $\mathrm{SO}(10)$ gauge symmetry.
} 
The scalar masses, then, can be written as

$$
\begin{aligned}
m_{H_{d}}^{2} & =m_{10}^{2}+2 D, \\
m_{H_{u}}^{2} & =m_{10}^{2}-2 D, \\
m_{Q, U, E}^{2} & =m_{16}^{2}+D, \\
m_{D, L}^{2} & =m_{16}^{2}-3 D .
\end{aligned}
$$

Such a contribution generically arises in the effective theory below the GUT scale as a consequence of spontaneous breakdown of the $\mathrm{U}(1)_{\mathrm{V}}$ at the GUT scale [9]. Finally, it is assumed that the soft trilinear couplings have a universal value $A_{0}$ at the GUT scale. ${ }^{5}$

It was shown in ref. [10] that for the universal gaugino masses the top-bottom-tau Yukawa unification can be realized only if $D>0$ and $m_{10}>m_{16}$. The same conditions are preferred in the $\mathrm{SO}(10)$ model studied in ref. [24] where the gaugino masses are given in eq. (2.1). Moreover, it was noted in ref. [24] that the Yukawa unification consistent with the REWSB requires specific correlations between parameters $D, m_{10}$ and $A_{0}$. Provided that these correlations hold, the top-bottom-tau Yukawa unification can be realized for a very wide range of $M_{1 / 2}$ and $m_{16}$. Non-trivial constraints on the model are obtained from the $\operatorname{BR}(b \rightarrow s \gamma)$, the $(g-2)_{\mu}$ and the upper bound on the relic abundance of the lightest neutralinos, $\Omega_{\mathrm{DM}} h^{2}$. Demanding that the predictions of the model for these observables are consistent with the experimental data, the assumption of Yukawa unification leads to rather definite prediction for the MSSM spectrum. This is mainly due to the tension between the $\mathrm{BR}(b \rightarrow s \gamma)$ and the $(g-2)_{\mu}$ : the former prefers heavy SUSY spectra while the latter the light ones. On top of that, the WMAP bound [47] on the $\Omega_{\mathrm{DM}} h^{2}$ can be satisfied only in some corners of the parameter space where various mechanisms which enhance the annihilation cross-section of the LSP are at work. It was found in ref. [24] that in the part of the parameter space where the Yukawa unification is consistent simultaneously with the $\operatorname{BR}(b \rightarrow$ $s \gamma)$ and the $(g-2)_{\mu}$, the gluino mass is predicted to have any value below about $1550 \mathrm{GeV}$. However, in the region of the parameter space where the gluino mass is below about $500 \mathrm{GeV}$ and between about 700 and $900 \mathrm{GeV}$, there is no efficient LSP annihilation mechanism that could reduce the thermal relic abundance of the neutralinos to acceptable values.

For the gluino mass between about 500 and $700 \mathrm{GeV}$ WMAP bound on the $\Omega_{\mathrm{DM}} h^{2}$ can be satisfied due to resonant annihilations of the LSP through the $Z$ boson or the lightest CP-even Higgs exchange. Throughout this paper we will refer to the region of the parameter space with the efficient LSP annihilation via $Z$ or $h^{0}$ as the light gluino region. In light of the recent LHC data it is not clear whether the model points with such a light gluino are still allowed. In ref. [43] ATLAS collaboration presented interpretation of the 0-lepton search in the simplified model in which all the sparticles except for the gluino, degenerate squarks of the first and second generations and the massless neutralino are decoupled. In such a model gluino with mass below $700 \mathrm{GeV}$ has been excluded at $95 \%$ C.L. for the squark masses up to $2 \mathrm{TeV}$, with the limit increasing to $1075 \mathrm{GeV}$ for equal mass

\footnotetext{
${ }^{5}$ This assumption requires the existence of a singlet F-term which dominates the soft trilinear couplings but gives a subdominant contribution to the gaugino masses. The phenomenology of models without the singlet F-term (and with the non-universal A-terms) will be investigated in a separate article.
} 
squarks and gluinos. In the light gluino region of the $\mathrm{SO}(10)$ model of ref. [24], the masses of $\tilde{u}_{R}, \tilde{u}_{L}$ and $\tilde{d}_{L}$ (which are degenerate to large extent) are in the range 1.1-1.6 TeV, while the mass of $\tilde{d}_{R}$ (which is always lighter than the other first generation squarks due to the $D$-term splitting of soft scalar masses) is between about 900 and $1300 \mathrm{GeV}$. Applying limits on the gluino and squark masses in the simplified model to that $\mathrm{SO}(10)$ model one would exclude the light gluino region. However, the limits on the gluino and squarks masses in the simplified model described above should be treated as an useful indication to what extent the gluino and squark masses can be constrained with the available LHC data. For realistic models, these limits will be typically weaker. One of the main purposes of the present paper is to check whether the light gluino region of the $\mathrm{SO}(10)$ model of ref. [24] is still allowed by the recent LHC data.

There are at least two reasons why the limits from the 0-lepton search on the squark and gluino masses in the $\mathrm{SO}(10)$ model of [24] may be substantially weaker than in the simplified model. First, the winos and Higgsinos are always lighter than the gluino at the EW scale. ${ }^{6}$ Therefore, the gluino usually decays to the chargino or neutralino other than the bino-like LSP, unlike in the simplified model where the gluino decays to $q \bar{q} \tilde{\chi}_{1}^{0}$ through an off-shell squark. Second, in some part of the parameter space the right-handed sbottom is lighter than the gluino. For such model points gluino decays exclusively to $\tilde{b}_{1}$ (associated with a bjet) which subsequently decays most of the times to the Higgsino-like neutralino or chargino unless these decays are kinematically inaccessible. Longer gluino decay chains result on average in smaller $p_{\mathrm{T}}$ of individual jets and such events are less likely to pass the selection cuts.

In the second region with the gluino mass between about 900 and $1550 \mathrm{GeV}$, three mechanisms enhancing the LSP annihilations may be at work: t-channel exchange of the right-handed sbottom, resonant annihilation through the CP-odd Higgs, $A^{0}$, and coannihilations with the stau. The first aforementioned mechanism requires the sbottom to be lighter than about $200 \mathrm{GeV}$. The Yukawa-unified solutions with such a light sbottom have been found in the numerical scan performed in ref. [24] but only in the part of the parameter space where the LSP mass is below $100 \mathrm{GeV}$. Such solutions are excluded at $95 \%$ C.L. by the D0 search at the Tevatron [48]. The Yukawa-unified solutions, in which the LSP annihilation via the $A^{0}$ exchange dominates the total annihilation cross-section, were ruled out very recently by the LHC direct $A^{0} \rightarrow \tau \tau$ searches. This follows from the fact that the LSP annihilation via the $A^{0}$ exchange in the $\mathrm{SO}(10)$ model of ref. [24] can be large enough only when $m_{A^{0}} \lesssim 400 \mathrm{GeV}$ and $\tan \beta$ between 45 and 50, while the ATLAS [49] and CMS [50] analyses excluded $m_{A^{0}}$ below $400(450) \mathrm{GeV}$ for $\tan \beta \approx 45$ (50) at $95 \%$ C.L.. The limits on the $\left(m_{A^{0}}, \tan \beta\right)$ plane do not depend significantly on the other MSSM parameters [51], and these limits are also applicable to that $\mathrm{SO}(10)$ model in a rather good approximation.

The regions of the parameter space where the WMAP bound is satisfied due to the stau coannihilation are not constrained by any experiment so far. The values of the gluino mass found in the stau-coannihilation region are between about 1000 and $1550 \mathrm{GeV}$, the masses of the $\tilde{u}_{L}$ and the $\tilde{d}_{L}$ are in the range $1.3-1.8 \mathrm{TeV}$, while the $\tilde{u}_{R}\left(\tilde{d}_{R}\right)$ are lighter by about 100 (300) GeV than the left-handed squarks. In contrast to the light gluino mass region,

\footnotetext{
${ }^{6} M_{3} \sim-2 M_{2} \sim-12 M_{1}$ at the EW scale.
} 
in the stau-coannihilation region, the gluino is always heavier than all the third generation squarks and often heavier than the $\tilde{d}_{R}$. Most importantly, the $\tilde{b}_{1}$ is always lighter than the gluino by at least $500 \mathrm{GeV}$ which implies that the sbottom production is comparable or even more significant than the gluino and first generation squarks production. Notice that the spectra corresponding to the stau-coannihilation region are too heavy to be excluded even if one applies the limits found in the simplified model with the gluino, the LSP and only the first generation squarks (based on the 0-lepton search) and the simplified model with the gluino, the LSP and the lighter sbottom (based on the b-jet search) which typically overestimate the true exclusion limits. Nevertheless, we include this region in our analysis to confirm this expectation.

There is also a part of the parameter space where both the $A^{0}$ exchange and the stau-coannihilation contribute to the LSP annihilation cross-section at similar rate and the WMAP bound is satisfied. In this region, the required $A^{0}$ mass has not been excluded by the current LHC searches. However, we should comment that such solutions require $m_{A^{0}}$ to be smaller than about $480 \mathrm{GeV}$, and those solutions may be excluded when the whole LHC data accumulated in 2011 are analysed. The gluino and squark masses corresponding to such solutions are similar to those for the stau-coannihilation region.

We should also comment on the fact that the only Yukawa-unified solutions corresponding to the gluino mass in the range $900-1000 \mathrm{GeV}$ are those with a very light sbottom or $m_{A^{0}} \lesssim 400$. As we pointed out above, the former are excluded by the Tevatron while the latter are excluded by the LHC. This is an interesting example of a case in which parameter $M_{1 / 2}$ and thus the gluino mass can be constrained not because of excessive production of the gluinos in the $p p$ collisions but as a consequence of some correlations between the MSSM parameters in a specific subclass of MSSM models.

Another interesting feature of the $\mathrm{SO}(10)$ model is the fact that the lightest CP-even Higgs is predicted to be below about $114.5 \mathrm{GeV}$ in the region of the parameter space where the $(g-2)_{\mu}$ and the $b \rightarrow s \gamma$ are simultaneously satisfied. This is dangerously close to the LEP2 bound [52] but taking into account the fact that the uncertainty of this prediction is about $3 \mathrm{GeV}$ [53] this region of the parameter space is still allowed by the experimental data. We should also emphasise that the lightest Higgs can be much heavier if the constraint from the $(g-2)_{\mu}$ is neglected. In particular, the mass of the Higgs can be about $125 \mathrm{GeV}$, as suggested by the excess observed recently by ATLAS [54] and CMS [55], but the corresponding masses of the squarks and the gluino are too heavy to be discovered at the LHC with $\sqrt{s}=7 \mathrm{TeV}$.

\section{ATLAS searches for squarks and gluinos}

In order to investigate the LHC constraints on the $\mathrm{SO}(10)$ model we focus in this paper on the SUSY searches performed by ATLAS, although one can expect that the limits from the CMS searches are comparable. The 0-lepton search performed by ATLAS [43] with $1.04 \mathrm{fb}^{-1}$ of data defines five signal regions with at least two, three or four jets. The main kinematic variables used in that analysis are the effective mass, $m_{\mathrm{eff}}$, and the magnitude of the missing transverse momentum $E_{\mathrm{T}}^{\text {miss }} \equiv\left|\vec{p}_{\mathrm{T}}^{\text {miss }}\right|$. The $m_{\mathrm{eff}}$ is defined as the sum of $E_{\mathrm{T}}^{\text {miss }}$ 


\begin{tabular}{|c|c|c|c|c|c|}
\hline Signal Region & $\geqslant 2$ jets & $\geqslant 3$ jets & $\geqslant 4$ jets low & $\geqslant 4$ jets high & High mass \\
\hline$E_{\mathrm{T}}^{\text {miss }}$ & $>130$ & $>130$ & $>130$ & $>130$ & $>130$ \\
Leading jet $p_{\mathrm{T}}$ & $>130$ & $>130$ & $>130$ & $>130$ & $>130$ \\
Second jet $p_{\mathrm{T}}$ & $>40$ & $>40$ & $>40$ & $>40$ & $>80$ \\
Third jet $p_{\mathrm{T}}$ & - & $>40$ & $>40$ & $>40$ & $>80$ \\
Fourth jet $p_{\mathrm{T}}$ & - & - & $>40$ & $>40$ & $>80$ \\
$\Delta \phi_{\mathrm{min}}$ & $>0.4$ & $>0.4$ & $>0.4$ & $>0.4$ & $>0.4$ \\
$E_{\mathrm{T}}^{\mathrm{miss}} / m_{\mathrm{eff}}$ & $>0.3$ & $>0.25$ & $>0.25$ & $>0.25$ & $>0.2$ \\
$m_{\mathrm{eff}}$ & $>1000$ & $>1000$ & $>500$ & $>1000$ & $>1100$ \\
\hline SM Background & $62.4 \pm 4.4 \pm 9.3$ & $54.9 \pm 3.9 \pm 7.1$ & $1015 \pm 41 \pm 144$ & $33.9 \pm 2.9 \pm 6.2$ & $13.1 \pm 1.9 \pm 2.5$ \\
Data & 58 & 59 & 1118 & 40 & 18 \\
\hline
\end{tabular}

Table 1. The cuts used to define the signal regions of the ATLAS 0-lepton analysis [43]. All the dimensionful quantities are given in GeV. The $\Delta \phi_{\min }$ is the minimum azimuthal angle between $\vec{p}_{\mathrm{T}}^{\text {miss }}$ and $\vec{p}_{\mathrm{T}}$ of the three leading jets. The number of events observed by ATLAS in each signal region, as well as the expected Standard Model background are also displayed. The first uncertainty represents the statistical uncertainty, while the second is the systematic one.

and the magnitudes of the transverse momentum, $p_{\mathrm{T}} \equiv\left|\vec{p}_{\mathrm{T}}\right|$, of two, three or four leading jets for the signal region with at least two, three or four jets, respectively. In the "high mass" signal region, however, the $m_{\mathrm{eff}}$ is defined as the sum of $E_{\mathrm{T}}^{\mathrm{miss}}$ and $p_{\mathrm{T}}$ of all jets with $p_{\mathrm{T}}>40 \mathrm{GeV}$. All the signal regions require high $p_{\mathrm{T}}$ jets and large $E_{\mathrm{T}}^{\text {miss }}$. The cuts applied in each signal region of the 0-lepton search are summarized in table 1.

In the $\mathrm{SO}(10)$ model of ref. [24], the right-handed sbottom is the lightest among the squarks. In some part of the parameter space, the sbottom is even lighter than the gluino. Therefore, one may expect that the sbottoms are significantly produced at the LHC, either directly from the proton-proton collisions or indirectly from the gluino decays. In consequence, a lot of b-jets are expected in a typical final state. That is why we use also the ATLAS b-jets 0-lepton analysis [22] with $0.83 \mathrm{fb}^{-1}$ of data to set constraints on that $\mathrm{SO}(10)$ model. In that analysis, four signal regions have been defined with the requirement of three or more jets and at least one or two b-jets, depending on the signal regions. In the ATLAS b-jet analysis the same kinematic variables as in the 0-lepton search are used. Since one of the main targets of the b-jet 0-lepton analysis is constraining models which predict light sbottoms, the cuts on the $m_{\text {eff }}$ are weaker than in the 0-lepton search. The cuts defining the four signal regions of the b-jet 0-lepton search are summarized in table 2 .

Another analysis which may be sensitive to the $\mathrm{SO}(10)$ model was released most recently and focuses on search for new phenomena with large jet multiplicities and missing transverse momentum [44] using $1.34 \mathrm{fb}^{-1}$ of data. Throughout this paper we will refer to that analysis as the multijets analysis. In that analysis events with leptons in the final state are discarded. Four different signal regions have been defined with the minimum number of jets varying between six and eight. For such a large number of jets, large trigger inefficiencies may occur. In order to maintain acceptable trigger efficiency a separation 


\begin{tabular}{|c|c|c|c|c|}
\hline Signal Region & $3 \mathrm{JA}$ & $3 \mathrm{JB}$ & $3 \mathrm{JC}$ & $3 \mathrm{JD}$ \\
\hline$E_{\mathrm{T}}^{\mathrm{miss}}$ & $>130$ & $>130$ & $>130$ & $>130$ \\
Leading jet $p_{\mathrm{T}}$ & $>130$ & $>130$ & $>130$ & $>130$ \\
Second jet $p_{\mathrm{T}}$ & $>50$ & $>50$ & $>50$ & $>50$ \\
Third jet $p_{\mathrm{T}}$ & $>50$ & $>50$ & $>50$ & $>50$ \\
Number of b-jets & $\geqslant 1$ & $\geqslant 1$ & $\geqslant 2$ & $\geqslant 2$ \\
$\Delta \phi_{\mathrm{min}}$ & $>0.4$ & $>0.4$ & $>0.4$ & $>0.4$ \\
$E_{\mathrm{T}}^{\text {miss }} / m_{\mathrm{eff}}$ & $>0.25$ & $>0.25$ & $>0.25$ & $>0.25$ \\
$m_{\mathrm{eff}}$ & $>500$ & $>700$ & $>500$ & $>700$ \\
\hline SM Background & $356_{-92}^{+103}$ & $70_{-22}^{+24}$ & $79_{-25}^{+28}$ & $13_{-5.2}^{+5.6}$ \\
Data & 361 & 63 & 76 & 12 \\
\hline
\end{tabular}

Table 2. The cuts used to define the signal regions of the ATLAS b-jets 0-lepton analysis [22].

\begin{tabular}{|c|c|c|c|c|}
\hline Signal Region & $7 \mathrm{j} 55$ & $8 \mathrm{j} 55$ & $6 \mathrm{j} 80$ & $7 \mathrm{j} 80$ \\
\hline Jet $p_{\mathrm{T}}$ & \multicolumn{3}{|c|}{$>55 \mathrm{GeV}$} & \multicolumn{2}{|c|}{$>80 \mathrm{GeV}$} \\
\hline$\Delta R_{j j}$ & \multicolumn{4}{|c|}{$>0.6$ for any pair of jets } \\
\hline Number of jets & $\geqslant 7$ & $\geqslant 8$ & $\geqslant 6$ & $\geqslant 7$ \\
\hline$E_{\mathrm{T}}^{\text {miss }} / \sqrt{H_{T}}$ & \multicolumn{5}{|c|}{$>3.5 \mathrm{GeV}^{1 / 2}$} \\
\hline SM Background & $39 \pm 9$ & $2.3_{-0.7}^{+4.4}$ & $26 \pm 6$ & $1.3_{-0.4}^{+0.9}$ \\
Data & 45 & 4 & 26 & 3 \\
\hline
\end{tabular}

Table 3. The cuts used to define the signal regions of the ATLAS multijets analysis [44].

$\Delta R_{j j}>0.6$ between all the jets with $p_{\mathrm{T}}$ above the threshold for a given signal region is required. The cut on $E_{\mathrm{T}}^{\text {miss }} / \sqrt{H_{T}}$ where $H_{T}$ is the scalar sum of the transverse momenta of all the jets with $p_{\mathrm{T}}>40 \mathrm{GeV}$ and $|\eta|<2.8$ implies that also in that search a large missing transverse energy is required. The cuts applied in each signal region of the multijets search are detailed in table 3 .

The primary target of the multijets analysis is to provide increased sensitivity to models with sequential decays to many strongly interacting particles. It therefore fits very well with the purpose of constraining the $\mathrm{SO}(10)$ model. The multijets analysis has already been proved to be useful in the context of the CMSSM for which constraints in the region where $m_{1 / 2} \ll m_{0}$ from multijets analysis are comparable (or even better if $m_{0}$ is between about 1 and $1.5 \mathrm{TeV}$ ) to the ones from the 0-lepton search [44].

For each signal region $i$, the ATLAS papers provide the information on the observed number of events $n_{\mathrm{o}}^{(i)}$, the expected number of the Standard Model background events $n_{\mathrm{b}}^{(i)}$ together with its error $\sigma_{\mathrm{b}}^{(i)}$. These numbers are given in tables 1, 2, 3. The agreement be- 
tween $n_{\mathrm{o}}^{(i)}$ and $n_{\mathrm{b}}^{(i)}$ can place constraints on SUSY models which predict the expected signal events $n_{\mathrm{s}}^{(i)}$ with its error $\sigma_{\mathrm{s}}^{(i)}$. In the next section we shall describe our procedure of computing $n_{\mathrm{s}}^{(i)}$ and $\sigma_{\mathrm{s}}^{(i)}$ and validate it against the ATLAS exclusion plots for the CMSSM (in the case of the 0-lepton [43] and the multijets [44] analyses) and the simplified model with the gluino, the sbottom and the lightest neutralino (in the case of the b-jet analysis [22]).

\section{Methodology and its validation}

Let us now discuss our methodology of simulating the ATLAS searches. We use various publicly available codes. First, we compute the SUSY spectra with SOFTSUSY [56] whose outputs are passed to SUSYHIT [57-59] which calculates branching ratios for all SUSY particles. We simulate SUSY events using Herwig++ [60] where the effects of parton shower, hadronization and underling events are taken into account. 10000 events are generated for each model point. In the simulation we include only production channels with at least one squark or gluino. We do not simulate direct pair productions of the neutralinos, the charginos or the sleptons since they are either negligible or the $p_{\mathrm{T}}$ of jets and the effective mass, $m_{\text {eff }}$, corresponding to such events are too small to pass the selection cuts. The detector simulation is done using Delphes [61] with the ATLAS detector card modified according to the ATLAS analyses, where in particular jets are defined using the anti- $k_{T}$ algorithm with $\Delta R=0.4$ and the b-jet tagging efficiency is set to $50 \%$. Finally, the total SUSY production cross-section, $\sigma_{\mathrm{NLO}}$, is calculated at next-to-leading order with PROSPINO [62-65].

In order to work out the expected number of SUSY events in the signal region $i, n_{\mathrm{s}}^{(i)}$, we apply the cuts detailed in tables 1, 2 and 3 to find their efficiency $\epsilon$ (i.e. the ratio of the number of events that survived the cuts to the total number of generated events). The $n_{\mathrm{s}}^{(i)}$ is given by $\sigma_{\mathrm{NLO}} \times \epsilon^{(i)} \times \mathcal{L}^{(i)} \times A^{(i)}$, where $A^{(i)}$ is a correction factor which accounts for the impact of additional data cleaning due to electronics failure in one of the calorimeters in the ATLAS detector during some period of data-taking. We take $A=0.9$ for the b-jet analysis and $A=0.95$ for the 0-lepton and the multijets analyses.

In order to estimate the exclusion limits we mimic the ATLAS analyses to the extent that is allowed by the limited amount of information provided in the ATLAS publications on those analyses. In particular, the CLs method [66] is used which employs a variable CLs defined by:

$$
C L s \equiv \frac{p_{\mathrm{s}+\mathrm{b}}}{1-p_{\mathrm{b}}}
$$

to set the exclusion limits on a model at $(1-C L s) \times 100 \%$ confidence level, which means a model is excluded at $95 \%$ C.L. if $C L s<0.05$. In the above definition $p_{\mathrm{s}+\mathrm{b}}$ is the p-value of the background plus signal hypothesis while $p_{\mathrm{b}}$ is the p-value of the background only hypothesis. Given the information $\vec{\Sigma}^{(i)}=\left(n_{\mathrm{s}}^{(i)}, n_{\mathrm{b}}^{(i)}, \sigma_{\mathrm{s}}^{(i)}, \sigma_{\mathrm{b}}^{(i)}\right)$ for each signal region $i$, the p-values are computed in the following way [67]:

$$
p_{\mathrm{s}+\mathrm{b}}\left(n_{\mathrm{o}}^{(i)}\right)=\sum_{n=0}^{n_{\mathrm{o}}^{(i)}} P\left(n \mid \vec{\Sigma}^{(i)}\right), \quad p_{\mathrm{b}}\left(n_{\mathrm{o}}^{(i)}\right)=\sum_{n=n_{\mathrm{o}}^{(i)}}^{\infty} P\left(n \mid \vec{\Sigma}^{(i)}\right)
$$




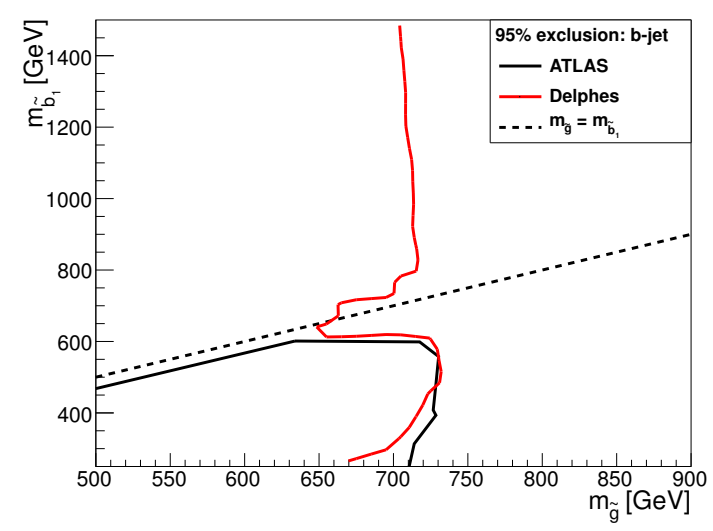

Figure 1. Comparison of our $95 \%$ exclusion contours with those of ATLAS in the simplified model in which only the gluino, the lighter sbottom and the LSP with mass of $60 \mathrm{GeV}$ are in the low energy spectrum, while all the other sparticles are decoupled. In contrast to the ATLAS exclusion contour, our cover also some region of the parameter space where $m_{\tilde{g}}<m_{\tilde{b}_{1}}+m_{b}$.

where $P\left(n \mid \vec{\Sigma}^{(i)}\right)$ is the probability of observing $n$ events in the signal region $i$ which is governed by Poisson distribution with the expectation value for the observed events:

$$
\lambda^{(i)}\left(\vec{\Sigma}^{(i)}, \delta_{b}, \delta_{s}\right)=n_{\mathrm{b}}^{(i)}\left(1+\delta_{b} \sigma_{\mathrm{b}}^{(i)}\right)+n_{\mathrm{s}}^{(i)}\left(1+\delta_{s} \sigma_{\mathrm{s}}^{(i)}\right),
$$

where the impact of the uncertainties on the expected number of events is accounted for by the nuisance parameters $\delta_{b}$ and $\delta_{s}$. We have neglected the error on the luminosity since it is negligible as compared to the errors we included. Assuming that the nuisance parameters have Gaussian probability distribution function, the probability of observing $n$ events is given by:

$$
P\left(n \mid \vec{\Sigma}^{(i)}\right)=\int_{-1 / \sigma_{s}^{(i)}}^{\infty} d \delta_{s} \int_{-1 / \sigma_{b}^{(i)}}^{\infty} d \delta_{b} \frac{e^{-\lambda^{(i)}}\left(\lambda^{(i)}\right)^{n}}{n !} e^{-\frac{1}{2}\left(\delta_{s}^{2}+\delta_{b}^{2}\right)}
$$

The lower limit of the integration is restricted to keep the number of signal and background events independently non-negative.

The ATLAS papers provide the error on the signal cross-section. It is estimated by varying the renormalization and factorization scales in PROSPINO between half and twice their default value and by considering the PDF uncertainties provided by CTEQ [68]. The resulting uncertainties on the signal cross-section vary somewhat from point to point in parameter space but it is reasonable to assume that the error on the cross-section for each production channel is constant across the parameter space. In our analysis we assign uncertainties of $10 \%, 20 \%, 30 \%, 35 \%$ for the $\tilde{q} \tilde{q}^{(*)}, \tilde{q} \tilde{g}, \tilde{g} \tilde{g}, \tilde{t} \tilde{t}^{*}$ and $\tilde{b}^{*}$ productions, respectively.

The ATLAS papers do not provide, however, the details on the other systematic uncertainties on the signal such as the jet energy scale uncertainty or the b-tagging uncertainty. We model these uncertainties by taking a constant value $\sigma_{\mathrm{s}^{\prime}}^{(i)}$ for the signal region $i$. Our estimation of the total systematic uncertainty on the signal is then given by $\sigma_{\mathrm{s}}^{(i)}=$ 
$\sqrt{\sigma_{\text {cross }}^{2}+\sigma_{\mathrm{s}^{\prime}}^{(i)^{2}}}$. In order to determine $\sigma_{\mathrm{s}^{\prime}}^{(i)}$ we adjust it manually in such a way that our 95\% C.L. exclusion contours match the official ones presented in the ATLAS publications.

We find that $\sigma_{\mathrm{s}^{\prime}}^{(3 J D)}=0.6$ reproduces the official ATLAS exclusion contours from the b-jet analysis [22] in the simplified model containing only the gluino, the sbottom and the lightest neutralino with the mass of $60 \mathrm{GeV}$ reasonably well, ${ }^{7}$ as seen from figure 1 . In the official ATLAS plot exclusion contours are restricted to the region of the parameter space in which the gluino mass is larger than the sum of the sbottom and bottom masses. In figure 1 we present extended version of that plot to the region of the parameter space where $m_{\tilde{g}}<m_{\tilde{b}_{1}}+m_{b}$ and the gluino decays through the off-shell sbottom into $b \bar{b} \tilde{\chi}_{1}^{0}$.

We also find that $\sigma_{\mathrm{s}^{\prime}}^{(2 j)}=\sigma_{\mathrm{s}^{\prime}}^{(3 j)}=\sigma_{\mathrm{s}^{\prime}}^{(4 j \text { high })}=0.3$ and $\sigma_{\mathrm{s}^{\prime}}^{(\mathrm{hm})}=\sigma_{\mathrm{s}^{\prime}}^{(3 j)}=\sigma_{\mathrm{s}^{\prime}}^{(4 j \text { low })}=0.1$ give a rather good approximation of the ATLAS exclusion contours for the 0-lepton search, as seen from figure 6 in the appendix. For the multijets analysis we find the best fit for $\sigma_{\mathrm{s}^{\prime}}^{(7 j 55)}=\sigma_{\mathrm{s}^{\prime}}^{(8 j 55)}=\sigma_{\mathrm{s}^{\prime}}^{(6 j 80)}=\sigma_{\mathrm{s}^{\prime}}^{(7 j 80)}=0.1$, as seen from figure 7 in the appendix.

\section{ATLAS constraints on the $\mathrm{SO}(10)$ model}

We are now ready to present resulting constraints on the $\mathrm{SO}(10)$ model from our simulation of the ATLAS analyses. We analysed 977 points (out of which 490 are in the light gluino region) satisfying the top-bottom-tau Yukawa unification at the level of $10 \%$ or better found in a numerical scan performed in ref. [24]. All of these points satisfy the following experimental constraints (which were computed with appropriate routines in MicrOmegas [69]):

$$
\begin{array}{rlrl}
12.7 \cdot 10^{-10} & <\delta a_{\mu}^{\mathrm{SUSY}}<44.7 \cdot 10^{-10}(2 \sigma) & & {[70,71]} \\
2.89 \cdot 10^{-4} & <\mathrm{BR}(b \rightarrow s \gamma)<4.21 \cdot 10^{-4}(2 \sigma) & & {[72-74]} \\
\mathrm{BR}\left(B_{s} \rightarrow \mu^{+} \mu^{-}\right) & <1.08 \cdot 10^{-8} & {[19]} \\
\Omega_{\mathrm{DM}} h^{2} & <0.1288 \quad(3 \sigma) & & {[47]} \\
m_{h^{0}} & >111.4 \mathrm{GeV} & & {[52]}
\end{array}
$$

Notice that we have included the recent constraint on the $\operatorname{BR}\left(B_{s} \rightarrow \mu^{+} \mu^{-}\right)$from the combined analysis of CMS and LHCb [19]. However, this observable is much less constraining than the lower limit on $m_{A^{0}}$ which we set in our analysis at $400 \mathrm{GeV}$ independently of $\tan \beta$ (which varies between about 45 and 50 in the region that might be affected by the present constraint on $m_{A^{0}}$ ). We use slightly relaxed bound on $m_{A^{0}}$ due to its (small) model dependence and the theoretical uncertainty in the prediction of $m_{A^{0}}$. The mass limits on SUSY particles from LEP and the Tevatron including the limit on the sbottom mass discussed in section 2 are also taken into account. Due to the fact that the uncertainty in the prediction of the lightest Higgs boson mass is about $3 \mathrm{GeV}$ [53], slightly relaxed LEP2 bound [52] is

\footnotetext{
${ }^{7}$ We do note include in our analysis the $3 \mathrm{JA}, 3 \mathrm{JB}$ and 3JC signal regions of the b-jet search because the exclusion plots for the individual signal regions has not been made publicly available so it was not possible to validate our simulation of these signal regions. We do not expect that inclusion of these signal regions would change our conclusions significantly since the 3JD signal region is the most constraining one in the vast majority of the parameter space [22].
} 


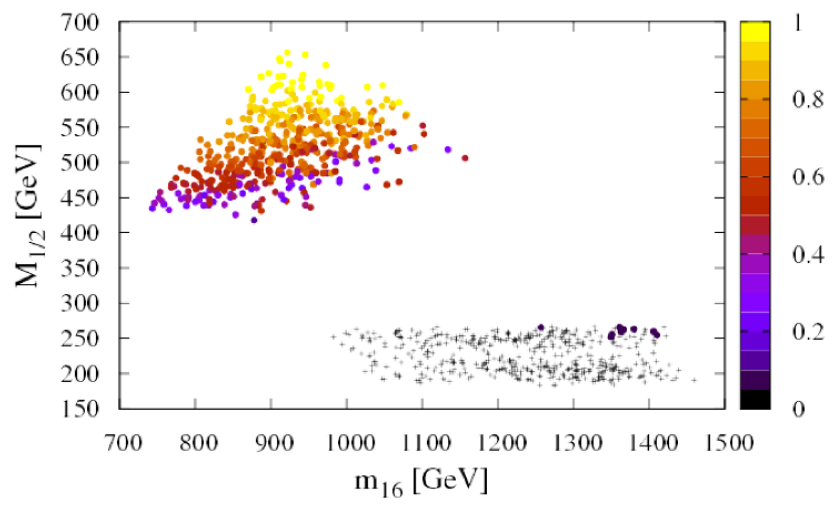

Figure 2. Distribution of the $C L s$ value in the $m_{16}-M_{1 / 2}$ plane based on the combination of the 0-lepton, the b-jet and the multijets searches. For each point in the plot, the signal region providing the smallest value of the $C L s$ is chosen. The black crosses correspond to the points excluded at $95 \%$ C.L..

\begin{tabular}{|c|c|c|c|c|c|c|c|c|c|c|c|c|}
\hline b-jet & \multicolumn{5}{|c|}{ 0-lepton } & \multicolumn{5}{c|}{ multijets } & \\
\hline 3JD & $2 \mathrm{j}$ & $3 \mathrm{j}$ & $4 \mathrm{j}$ low & $4 \mathrm{j}$ high & $\mathrm{hm}$ & $\mathrm{comb}$ & $7 \mathrm{j} 55$ & $8 \mathrm{j} 55$ & $6 \mathrm{j} 80$ & $7 \mathrm{j} 80$ & comb & comb \\
\hline 59.2 & 0 & 8.6 & 0 & 24.5 & 94.1 & 94.1 & 74.3 & 85.9 & 87.3 & 44.1 & 90 & 98.2 \\
\hline
\end{tabular}

Table 4. Percentage of the excluded points at $95 \%$ C.L. in the light gluino region by individual signal regions. "comb" in the 0-lepton (multijets) column correspond to a combination of all the signal regions within the 0-lepton (multijets) search, while "comb" in the last column corresponds to the combination of all three ATLAS searches.

used. ${ }^{8}$ Even though we require the model points to satisfy only the upper WMAP bound, many of them satisfy also the lower WMAP bound on the dark matter relic density.

The main result of our analysis is that 481 out of 490 points in the light gluino region are excluded at $95 \%$ C.L.. On the other hand, none of the points are excluded in the stau-coannihilation region. This can be seen from figure 2 where the distribution of the $C L s$ value in the $m_{16}-M_{1 / 2}$ plane based on the combination of all searches is presented. For each point in this plot, the signal region providing the smallest $C L s$ value is chosen. The model points depicted by the crosses are excluded at 95\% C.L..

\footnotetext{
${ }^{8}$ Very recently ATLAS [54] and CMS [55] announced their preliminary results of the SM Higgs searches with $5 \mathrm{fb}^{-1}$ of data. Due to downward fluctuation in the data ATLAS extended the lower limit on the SM Higgs boson mass to $115.5 \mathrm{GeV}$ at $95 \%$ C.L.. We do not take this bound into account because the Higgs boson production cross-section in the MSSM is usually smaller than in the SM due to the negative contribution from a stop loop to the gluon fusion production rate, while ATLAS excluded only $\sigma \gtrsim 0.9 \sigma_{\mathrm{SM}}$ for $m_{h} \approx 115 \mathrm{GeV}$.
} 


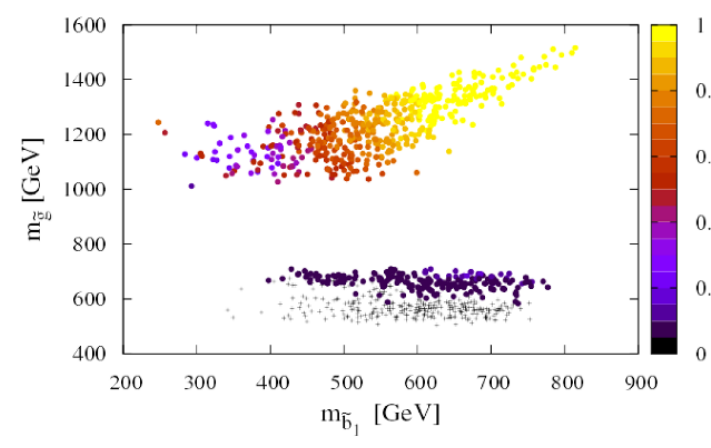

(a) b-jet

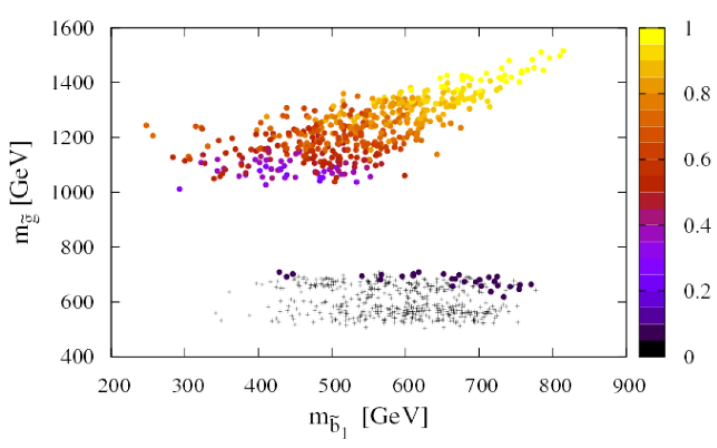

(b) 0-lepton

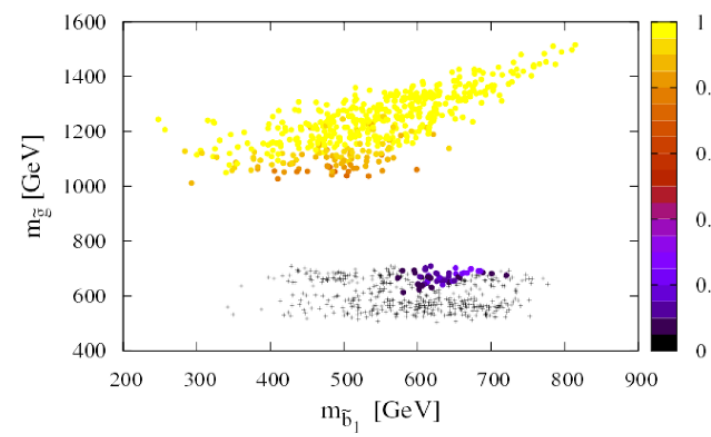

(c) multijets

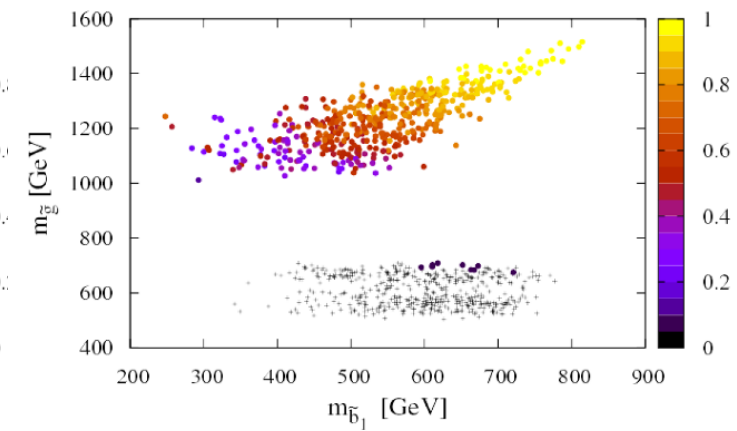

(d) combined

Figure 3. Distribution of the CLs value in the $m_{\tilde{b}_{1}}-m_{\tilde{g}}$ plane based on: (a) the b-jet search only, (b) the 0-lepton search only, (c) the multijets search only, (d) the combination of all searches. The black crosses correspond to the points excluded at 95\% C.L..

In figures 3 and 4, the distributions of the $C L s$ values based on the individual searches and their combination are shown in the $m_{\tilde{d}_{R}}-m_{\tilde{g}}$ and $m_{\tilde{b}_{1}}-m_{\tilde{g}}$ planes, respectively. The first thing to note from these figures is that the 0-lepton and the multijets analyses provide the most stringent constraints on the light gluino region but the b-jet search is only slightly less constraining ruling out by itself about $60 \%$ of points in this region. In table 4 , fractions of the excluded points in the light gluino region by individual signal regions are shown. In the 0-lepton analysis, essentially only the "high mass" region is relevant, while in the multijets analysis all the signal regions are relevant and complement each other. We should also emphasise the complementarity between the 0-lepton and the multijets searches. While individually these searches constrain the gluino mass to the values above about 620 and $610 \mathrm{GeV}$, respectively, their combination excludes all the points with the gluino mass below about $675 \mathrm{GeV}$. The multijets analysis is less sensitive to the part of the parameter space where $m_{\tilde{g}}>m_{\tilde{b}_{1}}$ and the mass splitting between the gluino and the 


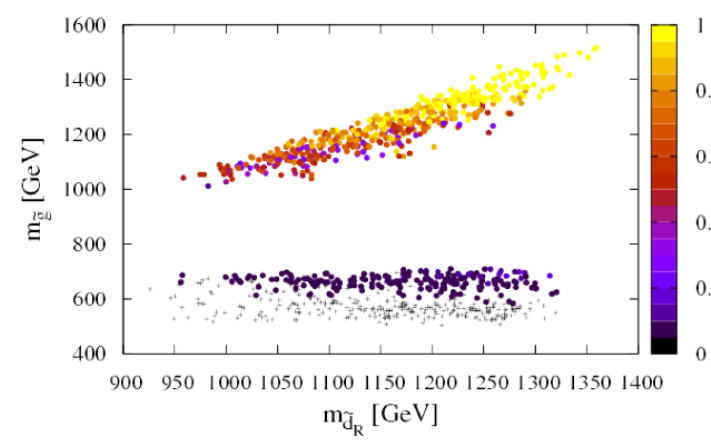

(a) b-jet

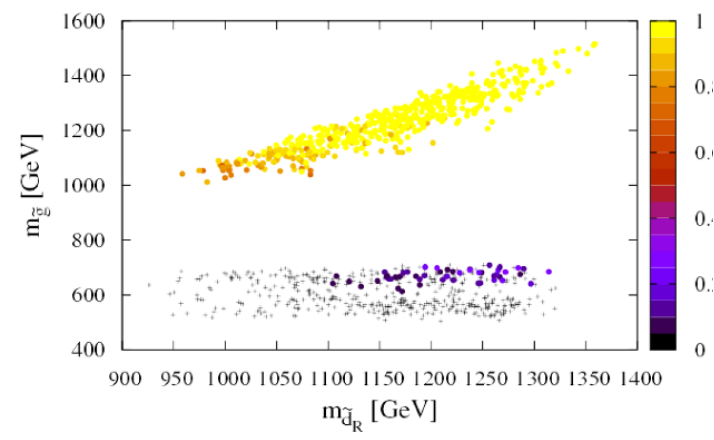

(c) multijets

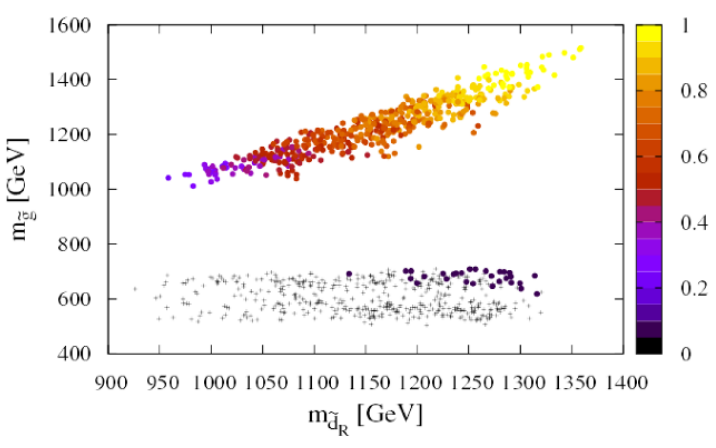

(b) 0-lepton

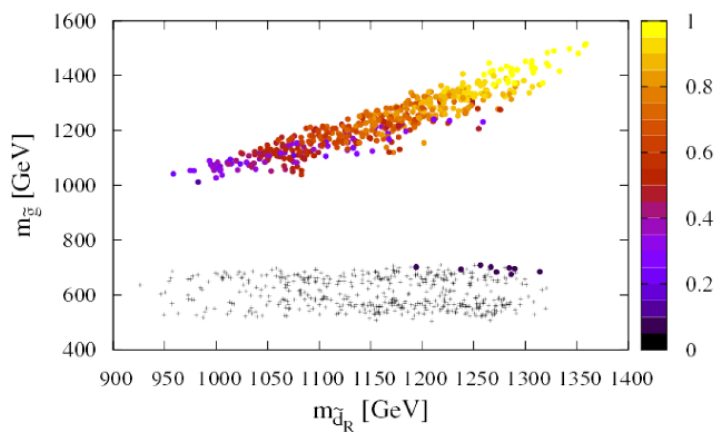

(d) combined

Figure 4. The same as in figure 3 but in the $m_{\tilde{d}_{R}}-m_{\tilde{g}}$ plane.

sbottom is small. In such a case the gluino to sbottom decay produces rather soft jets. This implies that passing the cuts with large jet multiplicities in such a case becomes less likely. ${ }^{9}$ Appearance of the soft jets in the final state affects also the efficiency of the "high mass" signal region of the 0-lepton search but in a milder way, so some of the points (but not all) with small mass splitting between the sbottom and the gluino which are not excluded by the multijets analysis are excluded by the 0-lepton search. As a result, all the points in the light gluino region which are still allowed are characterized by rather small mass splitting between the gluino and the sbottom - smaller than about $100 \mathrm{GeV}$, as can be seen from figure 3. Another feature of the points not excluded by any ATLAS search is rather heavy $\tilde{d}_{R}$. The lower bound on its mass in the light gluino region is found to be about $1.2 \mathrm{TeV}$, as clearly seen from figure 4.

\footnotetext{
${ }^{9}$ Note that our setup of the event simulation does not take account of the NLO matrix element correction with extra hard jets associated with the sparticle production. Including that contribution may slightly increase the efficiency for the events with soft jets from SUSY cascade decays. In this sense, our analysis provides a conservative limit.
} 
It is also interesting to note that all the model points in the light gluino region would be excluded if the ATLAS had not observed some small excess over the background (about $1 \sigma$ ) in the high mass signal region of the 0-lepton analysis (see the last column in table 1 ). Therefore, if the excess observed by ATLAS is just an upward fluctuation of the background, and not manifestation of SUSY events, the light gluino region will be excluded very soon.

Even though all of the points in the stau-coannihilation region are consistent with the present LHC data, one can draw some interesting conclusions from our analysis for this case too. First of all, the values of the CLs provided by the b-jet and the 0-lepton search are much smaller than those of the multijets search. This is because in the stau-coannihilation region the mass of the lighter sbottom is typically comparable to the Higgsino mass ${ }^{10}$ and the $\tilde{b}_{1} \rightarrow \tilde{\chi}_{1}^{-} t$ decay channel is closed. The sbottom decaying through this channel may produce as many as five jets if $\tilde{\chi}_{1}^{-}$decays subsequently to $\tilde{\chi}_{1}^{0} W$ so this channel being closed results in a big loss of the cuts efficiency in the multijets analysis. Even if the $\tilde{b}_{1} \rightarrow \tilde{\chi}_{1}^{-} t$ decay channel is open, as is the case for a small number of points in the stau-coannihilation region, the $\tilde{\chi}_{1}^{-}$usually decays to $\tilde{\nu}_{\tau} \tau$ or $\tilde{\tau}_{1} \nu_{\tau}$ rather than to $\tilde{\chi}_{1}^{0} W$ which results in leptons or at least a smaller number of hard jets in the final state making it unlikely that the events would pass the selection cuts used in the multijets analysis. Moreover, efficiency of the cuts used in the multijets analysis is reduced also by the fact that decays $\tilde{d}_{R}$ usually decays directly to the LSP producing only single jet.

The 0-lepton and the b-jet searches set complementary constraints on the staucoannihilation region. The b-jet search is the most sensitive (i.e. provides the smallest $C L s$ value) to model points with very light sbottom where the total SUSY cross-section is dominated by the sbottom production ${ }^{11}$ while the 0-lepton search provides the best constraints for models with light $\tilde{d}_{R}$, as seen from figures 3 and 4 .

We also estimate the expected constraints on the stau-coannihilation region in the $\mathrm{SO}(10)$ model with $10 \mathrm{fb}^{-1}$ of the integrated luminosity. The multijets search will not have enough sensitivity to exclude any points from this region, as expected from the above discussion. We found that the most promising for constraining the stau-coannihilation region is the 0-lepton search. It can be seen from figure 5 that the 0-lepton search can probe the gluino masses in the stau-coannihilation region up to $1.1 \mathrm{TeV}$ if the right-handed down squark is lighter than $1 \mathrm{TeV}$. In our estimation of the expected exclusions with $10 \mathrm{fb}^{-1}$, we rescale the statistical errors with the luminosity but keep the relative systematic error constant. This is certainly a conservative assumption. Thus the reach of the 0-lepton search will be even better. With this conservative assumption, the b-jet search will not be able to probe the stau-coannihilation region. However, if the systematic uncertainties on the background were to be reduced significantly, for example by data driven-methods of

\footnotetext{
${ }^{10}$ There are two reasons for this. One is that a requirement of the bottom-tau Yukawa unification positively correlates $|\mu|$ with the gluino mass. The second is that due to large negative contributions to the sbottom mass from $D$-term and the renormalization group effects (coming from a large positive $A_{0}$ required for consistency with the $b \rightarrow s \gamma$ constraint) the sbottom is light (see a detailed discussion in ref. [24]).

${ }^{11}$ In the final stages of preparing this work new ATLAS analysis was released [75] in which the limits on the $m_{\tilde{b}_{1}}-m_{\tilde{\chi}_{1}^{0}}$ plane were found that surpass those from D0. In that analysis $2 \mathrm{~b}$-jets are required in the final state and the cuts are optimized in order to maximise the sensitivity for models with the light sbottom. That ATLAS search excludes the model points in the stau-coannihilation region with the sbottom mass below about $350 \mathrm{GeV}$ if $\operatorname{BR}\left(\tilde{b}_{1} \rightarrow \tilde{\chi}_{1}^{0} b\right)=100 \%$ (which is usually the case in this part of the parameter space).
} 

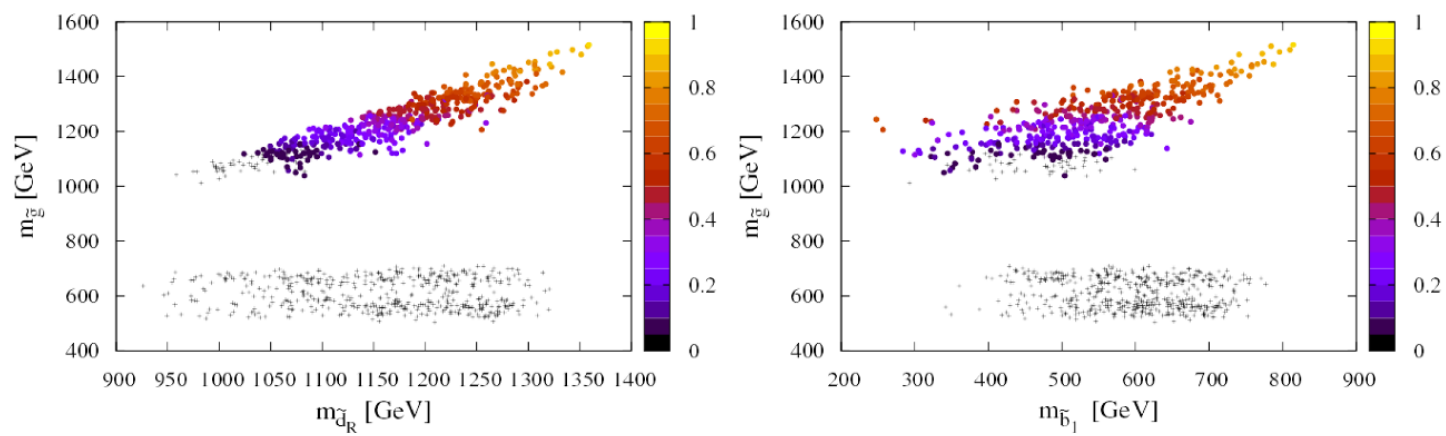

Figure 5. Distribution of the expected $C L s$ value based on the 0-lepton search with $10 \mathrm{fb}^{-1}$ of the integrated luminosity.

systematic error estimation, and/or the cuts were to be refined according to the larger amount of data, then the b-jet search may be complementary to the 0-lepton search, especially in the part of the parameter space with light sbottom.

\section{Summary and conclusions}

We have investigated the LHC constraints on the recently proposed SUSY SO(10) GUT model [24] predicting the top-bottom-tau Yukawa unification. In this model the non-universal gaugino masses arising from $\mathrm{SO}(10)$ non-singlet $F$-term transforming as $\mathbf{2 4}$ of $\mathrm{SU}(5) \subset \mathrm{SO}(10), D$-term splitting of the scalar masses and the negative sign of $\mu$ are assumed. The model is able to satisfy all experimental constraints including the $(g-2)_{\mu}$, the $b \rightarrow s \gamma$ and predict correct thermal relic abundance of the neutralinos. In the region of parameter space of the $\mathrm{SO}(10)$ model where all phenomenological constraints are fulfilled, relatively light MSSM spectra are predicted and there is a good prospect of discovering SUSY particles at the LHC. We found that this part of the $\mathrm{SO}(10)$ model parameter space tightened considerably due to the recent results of the LHC experiments which have not found any sign of SUSY so far, but many Yukawa-unified solutions are still consistent with all experimental data.

The phenomenologically viable parameter space of this model can be classified into three regions in terms of the dominant LSP annihilation process: the light gluino region (the dominant process is $Z$ boson or light CP-even Higgs exchange), the light $A^{0}$ region (the dominant process is CP-odd Higgs exchange) and the stau-coannihilation region (the dominant process is stau-coannihilation.). We have argued that the light $A^{0}$ region has already been excluded by the recent $A^{0} \rightarrow \tau \tau$ search at the LHC and we have investigated the impact of the recent direct SUSY searches on the light gluino and the stau-coannihilation regions in the $\mathrm{SO}(10)$ model.

Since the constraints on the gluino and squark masses obtained in the simplified model, where only the massless neutralino, the gluino and the first/second generation of squarks are assumed in a low energy SUSY spectrum, cannot be directly applied to other models, we performed a dedicated analysis to assess the impact of the recent direct 
SUSY searches on the SO(10) model. We focused on three ATLAS analyses: the 0-lepton search with $1.04 \mathrm{fb}^{-1}$ [43], the b-jet search with $0.83 \mathrm{fb}^{-1}$ [22] and the multijets search with $1.34 \mathrm{fb}^{-1}$ [44] of data. We found the $95 \%$ C.L. lower limit of $675 \mathrm{GeV}$ for the gluino mass, substantially weaker than the one obtained in the simplified model. This implies that the light gluino region is excluded except for the corner of the parameter space where the gluino mass is between $675 \mathrm{GeV}$ and $700 \mathrm{GeV}$, the right-handed down squark mass is above $1.2 \mathrm{TeV}$ and the mass splitting between the gluino and the lighter sbottom is smaller than about $100 \mathrm{GeV}$. We, however, stress that the light gluino region would be completely excluded in the absence of the slight excess of the events observed in the "high mass" signal region of the ATLAS 0-lepton search. Therefore, this region will be excluded very soon unless the observed excess is not just an upward fluctuation of the background.

We also found that the stau-coannihilation region remained unconstrained by the present searches. We have estimated the expected exclusion limit on this region assuming the same analyses but with $10 \mathrm{fb}^{-1}$ of the integrated luminosity at the $7 \mathrm{TeV}$ LHC and found that the 0-lepton search may set the $95 \%$ C.L. lower limit on the gluino mass at 1.1 TeV if the right-handed down squark is not heavier than about $1 \mathrm{TeV}$. The b-jet search may also contribute to the constraints on the stau-coannihilation region, especially in the part of parameter space with light sbottom, if the systematic error in that search is reduced and/or the selection cuts are refined.

In conclusion, the $\mathrm{SO}(10)$ model of ref. [24] is still consistent with all available direct and indirect constraints, with good prospects for testing it at the LHC in the near future. This should be contrasted with the Yukawa-unified SO(10) models with the universal gaugino masses where the most favourable part of the parameter space has already been excluded by the LHC experiments. For this reason we believe that the model we have studied in the present paper is the best motivated and the most promising $\mathrm{SO}(10)$ GUT model that predicts the top-bottom-tau Yukawa unification. Therefore, it is certainly worth further detailed investigation, both on the theoretical and the experimental side.

\section{Acknowledgments}

This work has been partially supported by STFC. We would like to thank A. Barr and the Cambridge SUSY Working group, particularly B. C. Allanach, B. Gripaios and C. Lester, for helpful discussions. MB would also like to thank M. Olechowski for inspiring discussions and S. Pokorski for discussions and comments on the manuscript. We are indebted to J. Gaunt for reading the first version of this paper and suggesting some refinements to the text.

\section{A Validation of our simulation}

In this appendix we present the validation of our simulation of the ATLAS 0-lepton and multijets searches. For each of the signal region in those searches we determine the $95 \%$ C.L. exclusion region in the $\tan \beta=10, A_{0}=0, \mu>0$ slice of the CMSSM and compare to the corresponding exclusion region obtained by ATLAS. Such comparisons are shown in figures 6 and 7 for the 0 -lepton search and the multijets search, respectively. It can be 


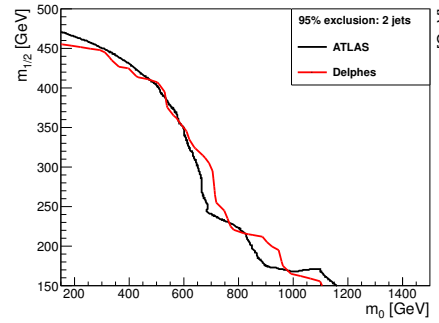

(a) 2 jets

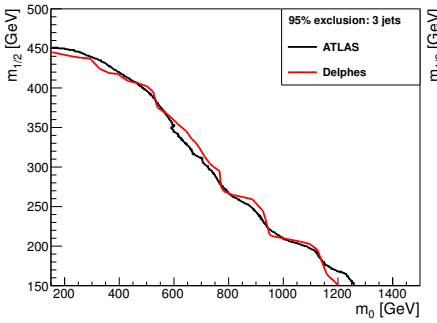

(b) 3 jets

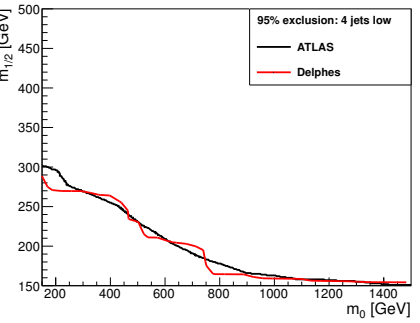

(c) 4 jets low

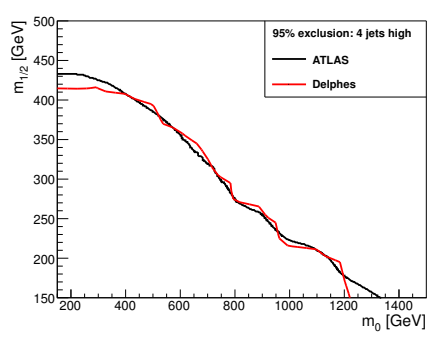

(d) 4 jets high

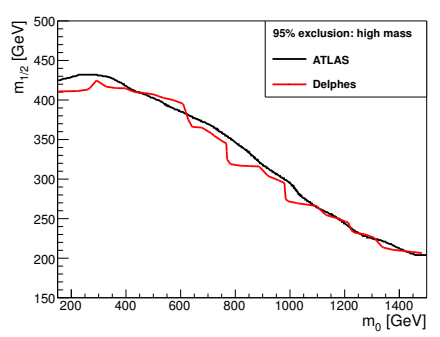

(e) high mass

Figure 6. Comparison of our 95\% C.L. exclusion contours with those of ATLAS in the $\tan \beta=10$, $A_{0}=0, \mu>0$ slice of the CMSSM for the 0-lepton search.

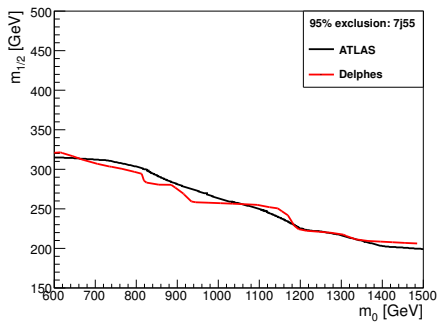

(a) $7 \mathrm{j} 55$

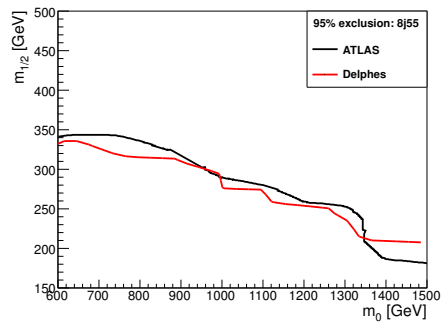

(b) $8 \mathrm{j} 55$

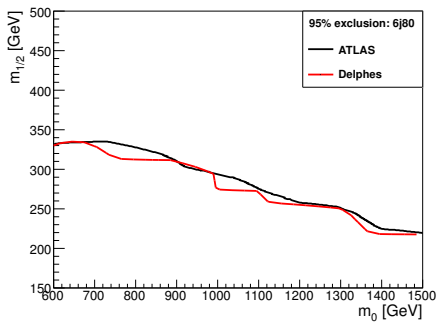

(c) $6 \mathrm{j} 80$

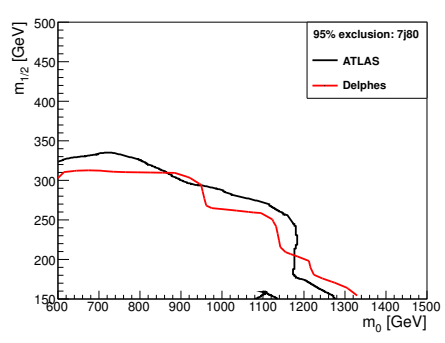

(d) $7 \mathrm{j} 80$

Figure 7. Comparison of our 95\% C.L. exclusion contours with those of ATLAS in the $\tan \beta=10$, $A_{0}=0, \mu>0$ slice of the CMSSM for the multijets search.

seen that, for each signal region, our exclusion contour is similar to the ATLAS one so we conclude that our approximation is reasonable. 
Open Access. This article is distributed under the terms of the Creative Commons Attribution License which permits any use, distribution and reproduction in any medium, provided the original author(s) and source are credited.

\section{References}

[1] B. Ananthanarayan, G. Lazarides and Q. Shafi, Top mass prediction from supersymmetric guts, Phys. Rev. D 44 (1991) 1613 [InSPIRE].

[2] B. Ananthanarayan, G. Lazarides and Q. Shafi, Radiative electroweak breaking and sparticle spectroscopy with $\tan \beta \simeq m_{t} / m_{b}$, Phys. Lett. B 300 (1993) 245 [INSPIRE].

[3] L.J. Hall, R. Rattazzi and U. Sarid, The top quark mass in supersymmetric $\mathrm{SO}(10)$ unification, Phys. Rev. D 50 (1994) 7048 [hep-ph/9306309] [INSPIRE].

[4] M.S. Carena, M. Olechowski, S. Pokorski and C. Wagner, Electroweak symmetry breaking and bottom-top Yukawa unification, Nucl. Phys. B 426 (1994) 269 [hep-ph/9402253] [INSPIRE].

[5] D.M. Pierce, J.A. Bagger, K.T. Matchev and R.-J. Zhang, Precision corrections in the minimal supersymmetric standard model, Nucl. Phys. B 491 (1997) 3 [hep-ph/9606211] [INSPIRE].

[6] G. D'Ambrosio, G. Giudice, G. Isidori and A. Strumia, Minimal flavor violation: an effective field theory approach, Nucl. Phys. B 645 (2002) 155 [hep-ph/0207036] [INSPIRE].

[7] C. Bobeth, T. Ewerth, F. Krüger and J. Urban, Enhancement of $B\left(\bar{B}(d) \rightarrow \mu^{+} \mu^{-}\right) / B\left(\bar{B}(s) \rightarrow \mu^{+} \mu^{-}\right)$in the MSSM with minimal flavor violation and large $\tan \beta$, Phys. Rev. D 66 (2002) 074021 [hep-ph/0204225] [INSPIRE].

[8] M. Olechowski and S. Pokorski, Electroweak symmetry breaking with nonuniversal scalar soft terms and large $\tan \beta$ solutions, Phys. Lett. B 344 (1995) 201 [hep-ph/9407404] [INSPIRE].

[9] Y. Kawamura, H. Murayama and M. Yamaguchi, Low-energy effective Lagrangian in unified theories with nonuniversal supersymmetry breaking terms, Phys. Rev. D 51 (1995) 1337 [hep-ph/9406245] [INSPIRE].

[10] H. Murayama, M. Olechowski and S. Pokorski, Viable t-b- $\tau$ Yukawa unification in SUSY SO(10), Phys. Lett. B 371 (1996) 57 [hep-ph/9510327] [InSPIRE].

[11] H. Baer, M.A. Diaz, J. Ferrandis and X. Tata, Sparticle mass spectra from $\mathrm{SO}(10)$ grand unified models with Yukawa coupling unification, Phys. Rev. D 61 (2000) 111701 [hep-ph/9907211] [INSPIRE].

[12] H. Baer et al., Yukawa unified supersymmetric $\mathrm{SO}(10)$ model: cosmology, rare decays and collider searches, Phys. Rev. D 63 (2000) 015007 [hep-ph/0005027] [INSPIRE].

[13] T. Blazek, R. Dermisek and S. Raby, Yukawa unification in SO(10), Phys. Rev. D 65 (2002) 115004 [hep-ph/0201081] [INSPIRE].

[14] D. Auto et al., Yukawa coupling unification in supersymmetric models, JHEP 06 (2003) 023 [hep-ph/0302155] [INSPIRE].

[15] H. Baer, S. Kraml, S. Sekmen and H. Summy, Dark matter allowed scenarios for Yukawa-unified SO(10) SUSY GUTs, JHEP 03 (2008) 056 [arXiv:0801.1831] [INSPIRE].

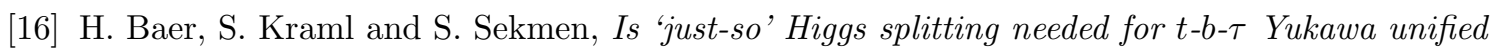
SUSY GUTs?, JHEP 09 (2009) 005 [arXiv:0908.0134] [INSPIRE]. 
[17] W. Altmannshofer, D. Guadagnoli, S. Raby and D.M. Straub, SUSY GUTs with Yukawa unification: a go/no-go study using FCNC processes, Phys. Lett. B 668 (2008) 385 [arXiv:0801.4363] [INSPIRE].

[18] S. Raby, Testing SUSY GUTs + family symmetry, talk given at Bethe Forum, http://www.bctp.uni-bonn.de/bethe-forum/2011/inaugural/talks/Raby-BF11.pdf, Bonn Germany november 2-18 2011.

[19] CMS and LHCB collaborations, Search for the rare decay $B_{s}^{0} \rightarrow \mu^{+} \mu^{-}$at the LHC with the $C M S$ and LHCb experiments: combination of LHC results of the search for $B_{s} \rightarrow \mu^{+} \mu^{-}$ decays, PAS-BPH-11-019, CERN, Geneva Switzerland (2011) [LHCb-CONF-2011-047] [CERN-LHCb-CONF-2011-047].

[20] H. Baer, S. Kraml, A. Lessa and S. Sekmen, Testing Yukawa-unified SUSY during year 1 of LHC: the role of multiple b-jets, dileptons and missing $E_{T}$, JHEP 02 (2010) 055 [arXiv:0911.4739] [INSPIRE].

[21] H. Baer, S. Raza and Q. Shafi, A heavier gluino from t-b- $\tau$ Yukawa-unified SUSY, arXiv: 1201.5668 [INSPIRE].

[22] ATLAS collaboration, Search for supersymmetry in pp collisions at $\sqrt{s}=7 \mathrm{TeV}$ in final states with missing transverse momentum, b-jets and no leptons with the ATLAS detector, ATLAS-CONF-2011-098, CERN, Geneva Switzerland (2011).

[23] M. Badziak, Yukawa unification in $\mathrm{SO}(10)$ in light of LHC data, to appear in Mod. Phys. Lett. A.

[24] M. Badziak, M. Olechowski and S. Pokorski, Yukawa unification in $\mathrm{SO}(10)$ with light sparticle spectrum, JHEP 08 (2011) 147 [arXiv:1107.2764] [INSPIRE].

[25] S.P. Martin, Non-universal gaugino masses from non-singlet F-terms in non-minimal unified models, Phys. Rev. D 79 (2009) 095019 [arXiv:0903.3568] [INSPIRE].

[26] U. Chattopadhyay and P. Nath, $b-\tau$ unification, $g_{\mu}-2$, the $b \rightarrow s+\gamma$ constraint and nonuniversalities, Phys. Rev. D 65 (2002) 075009 [hep-ph/0110341] [INSPIRE].

[27] M.J. Dolan, D. Grellscheid, J. Jaeckel, V.V. Khoze and P. Richardson, New constraints on gauge mediation and beyond from LHC SUSY searches at 7 TeV, JHEP 06 (2011) 095 [arXiv: 1104.0585] [INSPIRE].

[28] Y. Kats, P. Meade, M. Reece and D. Shih, The status of GMSB after $1 \mathrm{fb}^{-1}$ at the LHC, arXiv:1110.6444 [INSPIRE].

[29] B. Allanach, T. Khoo and K. Sakurai, Interpreting a $1 \mathrm{fb}^{-1}$ ATLAS search in the minimal anomaly mediated supersymmetry breaking model, JHEP 11 (2011) 132 [arXiv:1110.1119] [INSPIRE].

[30] S. Sekmen et al., Interpreting LHC SUSY searches in the phenomenological MSSM, arXiv:1109.5119 [INSPIRE].

[31] A. Arbey, M. Battaglia and F. Mahmoudi, Implications of LHC searches on SUSY particle spectra: the pMSSM parameter space with neutralino dark matter, Eur. Phys. J. C 72 (2012) 1847 [arXiv: 1110.3726] [INSPIRE].

[32] T.J. LeCompte and S.P. Martin, Large Hadron Collider reach for supersymmetric models with compressed mass spectra, Phys. Rev. D 84 (2011) 015004 [arXiv:1105.4304] [INSPIRE]. 
[33] T.J. LeCompte and S.P. Martin, Compressed supersymmetry after $1 \mathrm{fb}^{-1}$ at the Large Hadron Collider, arXiv:1111.6897 [INSPIRE].

[34] K. Sakurai and K. Takayama, Constraint from recent ATLAS results to non-universal sfermion mass models and naturalness, JHEP 12 (2011) 063 [arXiv:1106.3794] [INSPIRE].

[35] N. Bhattacharyya, A. Choudhury and A. Datta, Low mass neutralino dark matter in mSUGRA and more general models in the light of LHC data, Phys. Rev. D 84 (2011) 095006 [arXiv: 1107.1997] [INSPIRE].

[36] M. Papucci, J.T. Ruderman and A. Weiler, Natural SUSY endures, arXiv:1110.6926 [INSPIRE].

[37] C. Brust, A. Katz, S. Lawrence and R. Sundrum, SUSY, the third generation and the LHC, arXiv:1110.6670 [INSPIRE].

[38] R. Essig, E. Izaguirre, J. Kaplan and J.G. Wacker, Heavy flavor simplified models at the LHC, JHEP 01 (2012) 074 [arXiv:1110.6443] [INSPIRE].

[39] N. Desai and B. Mukhopadhyaya, Constraints on supersymmetry with light third family from LHC data, arXiv: 1111.2830 [INSPIRE].

[40] I. Gogoladze, R. Khalid, S. Raza and Q. Shafi, $t-b-\tau$ Yukawa unification for $\mu<0$ with a sub-TeV sparticle spectrum, JHEP 12 (2010) 055 [arXiv: 1008.2765] [INSPIRE].

[41] I. Gogoladze, R. Khalid and Q. Shafi, Yukawa unification and neutralino dark matter in $\mathrm{SU}(4)_{c} \times \mathrm{SU}(2)_{L} \times \mathrm{SU}(2)_{R}$, Phys. Rev. D 79 (2009) 115004 [arXiv: 0903.5204] [inSPIRE].

[42] M. Ajaib, T. Li and Q. Shafi, LHC constraints on NLSP gluino and dark matter neutralino in Yukawa unified models, Phys. Lett. B 705 (2011) 87 [arXiv:1107.2573] [INSPIRE].

[43] ATLAS collaboration, G. Aad et al., Search for squarks and gluinos using final states with jets and missing transverse momentum with the ATLAS detector in $\sqrt{s}=7 \mathrm{TeV}$ proton-proton collisions, arXiv:1109.6572 [INSPIRE].

[44] ATLAS collaboration, G. Aad et al., Search for new phenomena in final states with large jet multiplicities and missing transverse momentum using $\sqrt{s}=7 \mathrm{TeV}$ pp collisions with the ATLAS detector, JHEP 11 (2011) 099 [arXiv:1110.2299] [INSPIRE].

[45] I. Gogoladze, Q. Shafi and C.S. Un, Higgs boson mass from $t-b-\tau$ Yukawa unification, arXiv:1112.2206 [INSPIRE].

[46] I. Gogoladze, Q. Shafi and C.S. Un, SO(10) Yukawa unification with $\mu<0$, Phys. Lett. B 704 (2011) 201 [arXiv:1107.1228] [INSPIRE].

[47] WMAP collaboration, E. Komatsu et al., Seven-year Wilkinson Microwave Anisotropy Probe (WMAP) observations: cosmological interpretation, Astrophys. J. Suppl. 192 (2011) 18 [arXiv: 1001.4538 ] [INSPIRE].

[48] D0 collaboration, V.M. Abazov et al., Search for scalar bottom quarks and third-generation leptoquarks in pp collisions at $\sqrt{s}=1.96 \mathrm{TeV}$, Phys. Lett. B 693 (2010) 95 [arXiv: 1005.2222] [INSPIRE].

[49] ATLAS collaboration, Search for neutral MSSM Higgs bosons decaying to $\tau+\tau$-pairs in proton-proton collisions at $\sqrt{s}=7 \mathrm{TeV}$ with the ATLAS detector, ATLAS-CONF-2011-132, CERN, Geneva Switzerland (2011).

[50] CMS collaboration, Search for neutral Higgs bosons decaying to $\tau$ pairs in pp collisions at $\sqrt{s}=7 \mathrm{TeV}$, CMS-HIG-11-020, CERN, Geneva Switzerland (2011). 
[51] S. Gennai et al., Search for heavy neutral MSSM Higgs bosons with CMS: reach and Higgs-mass precision, Eur. Phys. J. C 52 (2007) 383 [arXiv:0704.0619] [INSPIRE].

[52] AlePh, Delphi, L3, OPAL, LeP Working Group for Higgs Boson Searches collaborations, S. Schael et al., Search for neutral MSSM Higgs bosons at LEP, Eur. Phys. J. C 47 (2006) 547 [hep-ex/0602042] [INSPIRE].

[53] B. Allanach, A. Djouadi, J. Kneur, W. Porod and P. Slavich, Precise determination of the neutral Higgs boson masses in the MSSM, JHEP 09 (2004) 044 [hep-ph/0406166] [INSPIRE].

[54] ATLAS collaboration, Combination of Higgs boson searches with up to $4.9 \mathrm{fb}^{-1}$ of $p p$ collisions data taken at a center-of-mass energy of $7 \mathrm{TeV}$ with the ATLAS experiment at the LHC, ATLAS-CONF-2011-163, CERN, Geneva Switzerland (2011).

[55] CMS collaboration, Combined results of searches for the standard model Higgs boson in pp collisions at $\sqrt{s}=7 \mathrm{TeV}$, CMS-HIG-11-032, CERN, Geneva Switzerland (2011) [arXiv: 1202.1488].

[56] B. Allanach, SOFTSUSY: a program for calculating supersymmetric spectra, Comput. Phys. Commun. 143 (2002) 305 [hep-ph/0104145] [INSPIRE].

[57] A. Djouadi, M. Muhlleitner and M. Spira, Decays of supersymmetric particles: the program SUSY-HIT (SUspect-SdecaY-Hdecay-InTerface), Acta Phys. Polon. B 38 (2007) 635 [hep-ph/0609292] [INSPIRE].

[58] A. Djouadi, J. Kalinowski and M. Spira, HDECAY: a program for Higgs boson decays in the standard model and its supersymmetric extension, Comput. Phys. Commun. 108 (1998) 56 [hep-ph/9704448] [INSPIRE].

[59] M. Muhlleitner, A. Djouadi and Y. Mambrini, SDECAY: a fortran code for the decays of the supersymmetric particles in the MSSM, Comput. Phys. Commun. 168 (2005) 46 [hep-ph/0311167] [INSPIRE].

[60] M. Bahr et al., HERWIG++ physics and manual, Eur. Phys. J. C 58 (2008) 639 [arXiv: 0803.0883] [INSPIRE].

[61] S. Ovyn, X. Rouby and V. Lemaitre, DELPHES, a framework for fast simulation of a generic collider experiment, arXiv:0903.2225 [INSPIRE].

[62] W. Beenakker, R. Hopker, M. Spira and P. Zerwas, Squark and gluino production at hadron colliders, Nucl. Phys. B 492 (1997) 51 [hep-ph/9610490] [InSPIRE].

[63] W. Beenakker, M. Krämer, T. Plehn, M. Spira and P. Zerwas, Stop production at hadron colliders, Nucl. Phys. B 515 (1998) 3 [hep-ph/9710451] [inSPIRE].

[64] M. Spira, Higgs and SUSY particle production at hadron colliders, hep-ph/0211145 [INSPIRE].

[65] T. Plehn, Measuring the MSSM Lagrangean, Czech. J. Phys. 55 (2005) B213 [hep-ph/0410063] [INSPIRE].

[66] A.L. Read, Presentation of search results: the CL(s) technique, J. Phys. G 28 (2002) 2693 [INSPIRE].

[67] B. Allanach, T. Khoo, C. Lester and S. Williams, The impact of the ATLAS zero-lepton, jets and missing momentum search on a CMSSM fit, JHEP 06 (2011) 035 [arXiv:1103.0969] [INSPIRE]. 
[68] P.M. Nadolsky et al., Implications of CTEQ global analysis for collider observables, Phys. Rev. D 78 (2008) 013004 [arXiv:0802.0007] [INSPIRE].

[69] G. Bélanger, F. Boudjema, A. Pukhov and A. Semenov, MicrOMEGAs 2.0: a program to calculate the relic density of dark matter in a generic model, Comput. Phys. Commun. 176 (2007) 367 [hep-ph/0607059] [INSPIRE].

[70] Muon G-2 collaboration, G. Bennett et al., Final report of the muon E821 anomalous magnetic moment measurement at BNL, Phys. Rev. D 73 (2006) 072003 [hep-ex/0602035] [INSPIRE].

[71] M. Davier, A. Hoecker, B. Malaescu and Z. Zhang, Reevaluation of the hadronic contributions to the muon $g-2$ and to $\alpha\left(M_{Z}^{2}\right)$, Eur. Phys. J. C 71 (2011) 1515 [arXiv: 1010.4180] [INSPIRE].

[72] Heavy Flavor Averaging Group collaboration, D. Asner et al., Averages of b-hadron, c-hadron and $\tau$-lepton properties, arXiv:1010.1589 [INSPIRE].

[73] M. Misiak et al., Estimate of $B\left(\bar{B} \rightarrow X_{s} \gamma\right)$ at $O\left(\alpha_{s}^{2}\right)$, Phys. Rev. Lett. 98 (2007) 022002 [hep-ph/0609232] [INSPIRE].

[74] G. Degrassi, P. Gambino and G. Giudice, $B \rightarrow X_{s} \gamma$ in supersymmetry: large contributions beyond the leading order, JHEP 12 (2000) 009 [hep-ph/0009337] [INSPIRE].

[75] ATLAS collaboration, Search for scalar bottom pair production with the ATLAS detector in pp collisions at $\sqrt{s}=7 \mathrm{Te} V$, arXiv:1112.3832 [INSPIRE]. 